\title{
Dinámicas socio-laborales en el Alto Valle de Río Negro: Transformaciones en la actividad frutihortícola
}

\author{
Social and labor dynamics in Alto Valle de Río Negro: transformations in fruit \\ farming
}

\author{
Fernando Svampa \\ Programa de Maestría en Ciencia, Tecnología e Innovación, \\ Universidad Nacional de Río Negro, Argentina \\ Contacto: correosyenviossvampa@gmail.com
}

\begin{abstract}
Resumen
La siguiente propuesta de trabajo pretende ahondar en torno a la actividad frutihortícola del Alto Valle de Río Negro en Argentina. A partir del análisis de las transformaciones en la estructura económica de la región y con el empleo de datos obtenidos de la Encuesta Permanente de Hogares y Censos Económicos se buscará dar cuenta por medio de ciertos indicadores de las condiciones de actividad de la población en el mercado laboral y los grupos poblacionales más afectados por las transformaciones de las últimas décadas en el sector, relacionadas con la modernización diferencial y la concentración económica. Se explorará la incidencia de estos procesos durante los años ulteriores a la devaluación del 2002 y su dinámica en los años posteriores hasta el 2013.
\end{abstract}

Palabras claves: Fruticultura, agroindustria, mercado de trabajo, tecnología.

\begin{abstract}
This article aims to contribute to a deeper understanding of fruit and vegetable farming in Alto Valle de Río Negro in Argentina. The argument is based on an analysis of economic structural changes in the region, alongside the use of data obtained from the Survey of Households and Economic Census. The objective is to discover, through certain indicators, the conditions of the labour market population and groups most affected by the transformations of recent d ecades i $n$ t he area, in relation to differentiated processes of modernization and economic concentration. The impact of these processes will be explored for the years after the devaluation of 2002 and its dynamics in the subsequent years until 2013.
\end{abstract}

Key words: Fruit farming, agro-industry, labor market, technology 
F. Svampa, Dinámicas socio-laborales en el Alto Valle de Río Negro.

Autoctonía. Revista de Ciencias Sociales e Historia, Vol. I, N²

\section{Introducción}

Los mercados de trabajo rural-urbanos se han caracterizado por una actividad primaria que ha marcado el ritmo productivo y la fisiología de una región más amplia que incluye áreas de poblaciones urbanas. Las actividades primarias están fuertemente condicionadas por la estacionalidad y los ciclos de producción que van moldeando el territorio en el cual lo rural y lo urbano se presentan como un continuo donde el campo persiste y el entorno guarda a menudo el encanto de lo rural, pero las formas de vida son predominantemente urbanas. Lo rural es un territorio distintivo y construido socialmente, producto del acceso, ocupación y de formas de dominación social que tienen como base material la estructura de tenencia, uso de la tierra y resultado también de la interacción campo-ciudad. El Alto Valle de Río Negro (de aquí en más AVRN) en el Norte de la Patagonia Argentina presenta estas características, con una actividad frutihortícola, especializada en la producción de pepita para exportación en fresco, mercado interno e industria, su dinámica ha condicionado no solo el asentamiento poblacional, sino el desarrollo de otros sectores productivos, urbanos y de infraestructura.

El área de cultivo frutícola constituye parte de los Departamentos de General Roca y Confluencia de las provincias de Río Negro y Neuquén ubicados en la región Norpatagónica. El AVRN está conformado por una larga franja de tierras, a partir de las oportunidades ecológicas que ofrecía el ecosistema, las poblaciones humanas intervinieron y modificaron el suelo para que recibieran el riego por gravitación y dieran comienzo a una etapa de cultivos, en particular la producción de forrajes y semillas de alfalfa a fines del siglo XIX. En las primeras décadas del siglo XX, la emergencia de una base social de pequeños y medianos productores familiares en el Alto Valle se dio en paralelo al rol del capital inglés en el desarrollo fundiario, transporte, la inserción de nuevas tecnologías y la comercialización almercado internacional. "Ese financiamiento provino de los propios productores, en base a su trabajo y del excedente que podían generar con sus primeros cultivos, y también, de la empresa monopólica del capital británico" (De Jong, 2010: 37). En el AVRN, la necesidad del cambio técnico representó una búsqueda constante por lograr mejores márgenes de eficiencia de la inelástica oferta de tierra cultivable, que constituyó la principal restricción productiva para el crecimiento económico.
El objetivo de este trabajo es ahondar acerca de las dinámicas socio-laborales en torno a la actividad frutihortícola del aglomerado rural-urbano Alto Valle de Rio Negro, particularmente en la situación de los chacareros y los trabajadores rurales. Para eso, el primer apartado describe el proceso de consolidación dela actividad frutihortícola en la región. En el segundo apartado se indagará sobre las transformaciones en la estructura económica en el aglomerado a partir de la reestructuración agroindustrial, en este sentido la diversidad de transformaciones en la producción y el empleo, pueden interpretarse desde la perspectiva de los cambios técnicos que impactan en la agroindustria, aumentando la productividad del trabajo en forma continua y modificando las posiciones laborales. El tercer apartado hará uso de fuentes secundarias estadísticas para analizar las condiciones de actividad de la población en el mercado laboral y los grupos poblacionales más afectados por las transformaciones de las últimas décadas en el sector, relacionadas con la modernización diferencial y la concentración económica. Se explorará la incidencia de estos procesos durante los años ulteriores a la devaluación del 2002 en la Argentina y su posterior dinámica hasta el 2013.

\section{Proceso de consolidación de un perfil productivo en el Alto Valle de Río Negro}

Diversas investigaciones regionales, algunas de ellas empleadas como soporte para este texto (Bandieri y Blanco, 1994; Bendini y Tsakoumagkos, 1999, 2002, 2003; Alvaro, 2013) coinciden en una determinada periodización de la actividad económicas en el AVRN.1

Un primer momento en la historia de la región, se caracterizó por el asentamiento poblacional y la conformación del perfil socio-productivo entre 18861930. Esta etapa se destacó por una colonización concentrada en un inicio, y posteriormente una colonización agraria capitalista expandida. El conjunto de transformaciones operadas a lo largo del siglo XIX estuvo dirigido a la organización de un modelo productivo y al cumplimiento de los requerimientos internaciones. Algunas de estas medidas están relacionadas con la consolidación de la propiedad privada de la tierra; el conjunto de leyes de regulación de la fuerza de trabajo; el proceso de mestización y mejoramiento de pasturas y razas animales; expansión 
F. Svampa, Dinámicas socio-laborales en el Alto Valle de Río Negro.

Autoctonía. Revista de Ciencias Sociales e Historia, Vol. I, N²

del ferrocarril entre otras. Este proceso se desenvolvió de formas distintas en el territorio nacional (Reguera, 2007). A fines de las últimas dos décadas del siglo XIX, el Estado argentino comenzó las obras de riego lo cual constituyó una acción fundamental para consolidar el perfil agrario. El primer canal fue construido en 1884, cobró impulso con la llegada del ferrocarril de la empresa Ferrocarril del Sud de capital británico. A partir de estas obras, la división en parcelas y la venta del suelo fue configurando el espacio vital y productivo, y otorgando la especificidad productiva al Valle (Blanco, 1999). El objetivo de la empresa Ferrocarril del Sud fue incrementar el tráfico ferroviario, como así también promover el desarrollo de infraestructura necesaria para encarar los procesos de configuración productiva. A partir de 1913 la empresa británica creó la Compañía de Tierras del Sud que funcionaba en la Estación Agronómica de Cinco Saltos -provincia de Río Negro- y organizó una cadena de comercialización a través de la Argentine Fruit Distributors (AFD) en 1928. La compañía británica Tierras del Sud promovió la subdivisión de tierras en pequeñas explotaciones y la figura inicial de este proceso fue el colono que participó en la adquisición de las tierras subdivididas en parcelas de 2 a 50 hectáreas (con fuerte participación de 10ha), (Bendini y Tsakoumagkos, 2003).

Un segundo momento en la historia de la región corresponde a la consolidación de la fruticultura entre 1930-1950, con predominio del capital monopólico inglés (en el sector de empaque, comercialización, tarifas ferroviarias, difusión de innovaciones tecnológicas) y de los chacareros como productores frutihortícolas. La organización social del territorio se desarrolló en base a la pequeña y mediana explotación y para fines de la década del '30 se constituyó la forma agrícola de producción intensiva de fruta pepita y la exportación a gran escala. En la región, los procesos por los que se dio la apropiación diferencial del suelo y su incorporación al mercado de tierras han consolidado durante el siglo XX una matriz productiva inicial de tipo farmer (Bendini y Tsakoumagkos, 2004). El término chacarero en esta zona alude a un productor que tradicionalmente combinó la propiedad de un pequeño monte frutal, el trabajo familiar y el uso de trabajadores transitorios y permanentes e inicialmente facilitó el desarrollo de la fruticultura, aunque con el proceso de modernización tecnológica se encontró limitado en sus opciones de expansión (De Jong, 2010). Durante este período se dio un crecimiento de la actividad regional a partir de los aumentos de producción y demanda externa (Bandieri y Blanco, 1994) a lo cual se agregó la escasez de oferta de fuerza laboral en el mercado de trabajo local, el agotamiento agrícola por el limitado alcance del sistema de riego; estos factores fortalecieron la impronta frutícola con un productor de dedicación exclusiva a la actividad, con participación del trabajo familiar y contratación de fuerza de trabajo complementaria en la explotación y la generación de excedentes (Miranda, 1995). Según fuentes oficiales, el Censo Nacional Agropecuario(CNA) de 1937 registraba que el $57 \%$ de las personas ocupadas en explotaciones agropecuarias en el departamento de General Roca en Río Negro, eran trabajadores familiares, la misma categoría se repite en los ocupados en Confluencia -provincia de Neuquén- que asciende al 63\% (CEIL/ PISPAL, 1982). Otra cuestión a tener presente es que en las décadas del ' 50 y ‘ 60 el requerimiento de mano de obra atrajo durante las épocas de cosecha, población de origen chileno y de otras partes del norte del país. Esos flujos migratorios, estacionales se mantuvieron hasta fines de los años ochenta (Aguilera, 2007).

A pesar de las dificultades estructurales relacionadas a la reconversión tecnológica y la persistente inflación, gran parte de los pequeños productores lograron perpetuar el uso de su unidad productiva y lograron acceder a la compra de nuevas parcelas en un marco de políticas públicas favorables, el desarrollo de la red vial y los avances en la industria frigorífica. Sin embargo, los productores no lograron una mejora en la participación del excedente total generado por la actividad, ni una mayor autonomía respecto a los empacadores/comercializadores (De Jong, et al., 1994)

Un tercer momento se ubicaría desde los años 19601970 y estaría caracterizado por la diferenciación del eslabón primario por integración agroindustrial, predominio del capital nacional oligopsónico en un contexto de modernización tecnológica generalizada en las cadenas de producción. Los principales protagonistas de esta etapa fueron los productores y los agentes locales que disponían de plantaciones, galpones y empacadores. La conformación del complejo agroindustrial frutícola se dio en paralelo al crecimiento de las exportaciones en términos 
F. Svampa, Dinámicas socio-laborales en el Alto Valle de Río Negro.

Autoctonía. Revista de Ciencias Sociales e Historia, Vol. I, N²

absolutos y relativos hacia mercados como Brasil y Estados Unidos. Esta fase de la fruticultura en el AVRN tuvo impactos diferenciales al interior de la estructura agraria. Los cambios en los mecanismos regionales de acumulación del sector empacadorcomercializador se expresaron en nuevas estrategias empresariales, como sucedió con la inserción en la producción primaria de estos actores, lo que dificultó el acceso a nuevas tecnologías para el resto de los eslabones de la actividad. Paulatinamente se fue quebrando el esquema de inversión productiva, configurando un paisaje social caracterizado por la fuerte diferenciación y heterogeneidad de los diversos sujetos sociales (trabajadores, chacareros, fruticultores), la pérdida de posición y de capacidad de negociación de los chacareros en un marco de concentración de los excedentes de la actividad en los eslabones de postcosecha (Bendini, 2007).

"Posteriormente al consolidarse el complejo agroindustrial y fortalecerse las tendencias expansivas de la actividad, se produce un incremento de la demanda de mano de obra permanente y se diversifica y amplía la movilidad espacial de la mano de obra estacional. También en esta etapa, se profundiza la diferenciación de la mano de obra asalariada en trabajadores rurales y, por otra parte, en operarios de galpones, frigoríficos e industrias de jugos y deshidratado, cuya expresión son las organizaciones gremiales con dinámicas propias y distintos niveles de negociación" (Tsakoumagkos y Bendini, 2002:2).

ElCNAde1960dacuentadeestas transformaciones, del total de ocupados en explotaciones agropecuarias, la proporción de trabajadores familiares de productores desciende al 39\% en Confluencia y al 34\% en General Roca. Por otro lado, se manifiesta un aumento de la participación de los asalariados permanentes, pasando estos del $10 \mathrm{al} 30 \%$ en Confluencia y del $15 \mathrm{al}$ 40\% en General Roca (CEIL/PISPAL, 1982).

Un cuarto momento está caracterizado por la modernización diferencial, acompañado por un incremento de la movilidad del capital y el trabajo (Bendini, 2007) y posibilidades asimétricas de adaptación tecnológica (Bendini y Tsakoumagkos, 2003). Durante esta etapa que inicia a fines de la década del ' 80 y principio de los ' 90 , que se extiende hasta el presente, se profundizaron las desigualdades entre los actores de la estructura agrícola valletana que participan de la actividad, en paralelo a una mayor subordinación-vulnerabilidad para el eslabón primario. La presencia del capital transnacional se remarcó en esta etapa, al igual que las exigencias del mercado (requisitos de calidad y sanidad) que repercutieron en los chacareros que adoptaron diversas estrategias adaptativas o de resistencia (Alvaro, 2013).

\section{Reestructuración del complejo agroindustrial frutícola}

El complejo agroindustrial frutícola puede ser interpretado en el marco de las tendencias de la agricultura en el contexto nacional y regional y comprende a un conjunto económico constituido por la división de las etapas de producción vinculadas a la transformación de las materias primas, cuya producción se basa en el control del potencial biológico en un espacio físico definido (Bendini y Pescio, 1996). En este sentido, la presencia de las empresas transnacionales es una constante en el proceso de agroindustrialización (Bendini y Pescio, 1996). El cambio técnicon en el complejo agroindustrial del AVRN se caracterizó por distintas etapas e lo largo de su proceso histórico.

Una primera etapa se observa durante el momento de conformación agroindustrial por integración hacia adelante a principios de 1960. Los principales protagonistasfueronlasempresaslocalesquedisponían de plantaciones y galpones en las mismas chacras, donde también embalaban fruta de otros productores. Los cambios tecnológicos más importantes de esta etapa fueron los vinculados a la mecanización y la incorporación de frigoríficos. La mecanización en chacra se manifestó en la incorporación del tractor en 1960, utilizado para los trabajos agrícolas como así también para el movimiento de insumos y productos; el uso de insecticidas orgánicos y poda americana fueron cambios técnicos significativos en este periodo (Merli y Bonifacio, 1996). El otro aspecto importante de esta etapa es la masiva construcción de frigoríficos y la consecuente integración entre el empaque y la conservación de la fruta. El salto cualitativo que significó este cambio técnico3 se vio reflejado en la incorporación de reglamentaciones más exigentes sobre conservación de la producción.

Una segunda etapa es la diferenciación agroindustrial interna, caracterizada por laincorporación de paquetes tecnológicos en chacra asociadas a los nuevos sistemas de conducción (espaldera y compacto), 3 cambios cualitativos en la manipulación y conservación de la fruta. 
F. Svampa, Dinámicas socio-laborales en el Alto Valle de Río Negro.

Autoctonía. Revista de Ciencias Sociales e Historia, Vol. I, N²

"Los nuevos sistemas de conducción se caracterizan por aumentar las dosis (absoluta y relativa) y la velocidad de circulación del capital. Al mismo tiempo modifican las labores culturales (demandan una pluralidad de nuevos procedimientos de poda basados en el raleo selectivo y en la ubicación de los frutos con gran accesibilidad para el trabajo), permiten mejorar la calidad de la producción y facilitan las tareas mecanizadas." (Tsakoumagkos y Bendini, 2002: 3).

Una tercera etapa se caracteriza por la concentración y transnacionalización, con cambios varietales en la producción primaria, en paralelo a nuevas técnicas agronómicas, como la automatización del empaque y conservación de la fruta inducidos desde la demanda y se profundizaron con la penetración del capital trasnacional. En la producción agrícola, los cambios técnicos en el complejo agroindustrial profundizaron la diferenciación tanto en los trabajadores permanentes como en los transitorios. Por un lado, se fue configurando un trabajador rural permanente central y más polivalente, de habilidades extensiva y semicalificado en las unidades productivas reconvertidas de mediano o gran tamaño (Bonifacio, 1996; Tsakoumagkos y Bendini, 2002). La disminución de la estacionalidad de algunas tareas que requieren cierta calificación ha permitido el surgimiento de lo que puede denominarse un trabajador permanente discontinuo (o transitorio permanente) ya que se prolonga el lapso de contratación respondiendo a la estrategia empresarial de optimizar la calidad con la flexibilización laboral (Tsakoumagkos y Bendini, 2003). En la producción industrial la diferenciación interna está relacionada con la calificación; al profundizarse la incorporación de tecnologías automáticas y electrónicas en empaque y frío que aumentan el ritmo, la intensidad del trabajo con nuevos requerimientos de calificación, se modifican y/o surgen nuevas posiciones laborales y en conjunto disminuye el volumen de trabajadores y aumenta la desestacionalización del trabajo (Tsakoumagkos y Bendini, 2002).

La modernización de la agricultura a partir de la década del '80 transformó las relaciones de producción al interior del sector, subordinando segmentos del circuito económico a las necesidades de expansión de empresas transnacionales agroindustriales. Por lo tanto, al analizar los aspectos de la modernización tecnológica, varios autores (Miranda, 1997; Bendini y Tsakoumagjos, 2001, Sacroisky, et. al., 2003) identifican que en un contexto, en el que el productor independiente no tiene disponibilidad de capital líquido, capacidad de endeudamiento para introducir innovaciones tecnológicas, riego por aspersión en la lucha contra heladas, o renovar variedades va reduciendo las labores culturales. Esta situación afecta la calidad de la fruta, se va generalizando la situación de pérdida de confianza en la viabilidad de su producción y el abandono del trabajo en los predios frutales, optando por el alquiler de la unidad productiva, o por la venta para loteo y construcción residencial si está próxima al núcleo urbano.

"En este marco, la revitalización del rol del capital fragmentado aparece como una nueva opción, bajo la forma denominada de "agricultura de contratos", en la cual el productor aparece totalmente "regulado" por el empacadorcomercializador, quien fija su precio, entrega insumos, realiza el control técnico, le brinda apoyo financiero y recibe la producción con un precio indicativo "conversado" (Sacroisky, 2003:20).

Como se dijo anteriormente, las grandes empresas, incorporaron la producción primaria en grandes explotaciones, modernas, capitalizadas, orientadas a la exportación en fruta fresco. Resulta que quienes ofrecen condiciones óptimas de producción, son aquellos que poseen 15 hectáreas destinadas a la fruticultura, que han podido capitalizarse y amoldarse a los requerimientos del mercado (Miranda, 1997; Sacroisky, 2003).

\section{Cambios técnicos en la producción primaria, sector de empaque y frigorífico}

Los cambios impuestos por la modernización económica supusieron en términos de dinámica económica y mercados globales, aumento de los ritmos, variedades y precios, exigencias de calidad; desde el punto de vista de la dinámica social y cultural, se alerta sobre la pérdida de oficio del chacarero, la ausencia de recambio generacional en los productores independientes; la menor transferencia de habilidades y competencias relacionadas con saberes que se transforman en obsoletos, volviendo a los productores dependientes de la capacidad de reconversión y actualización tecnológica (Landriscini, 2001). Con el objetivo de ilustrar el proceso de reconversión tecnológica en la producción primaria en las provincias de Río Negro y Neuquén, se comparte a continuación algunos indicadores que surgen de los Censos Nacionales Agropecuarios de 1988, 2002 y el Censo de Agricultura de Bajo Riego del 2005 en Río Negro. 
F. Svampa, Dinámicas socio-laborales en el Alto Valle de Río Negro.

Autoctonía. Revista de Ciencias Sociales e Historia, Vol. I, N²

Cuadro $\mathbf{N}^{\circ} 1$. Superficie implantada de las EAP con frutales de pepita (peras y manzanas), cantidad de plantas y cantidad de plantas por hectárea, 1988-2002. Río Negro y Neuquén.

\begin{tabular}{|l|l|l|l|l|l|l|}
\hline \multirow{2}{*}{ Río Negro y Neuquén } & \multicolumn{5}{l}{ Frutales de pepita } \\
\cline { 2 - 7 } & \multicolumn{5}{|l|}{ Total* } & \multicolumn{4}{l|}{ Manzano } & \multicolumn{3}{l|}{ Peral } \\
\cline { 2 - 7 } Río Negro & 1988 & 2002 & 1988 & 2002 & 1988 & 2002 \\
\hline Hectáreas & $42.174,60$ & $36.085,70$ & $30.991,30$ & $21.290,60$ & $10.728,40$ & $14.694,30$ \\
\hline Cantidad de Plantas & 12.980 .147 & 22.693 .240 & 9.193 .746 & 12.873 .809 & 3.648 .167 & 9.774 .294 \\
\hline Plantas/Has & 307,8 & 628,9 & 296,7 & 604,7 & 340 & 665,2 \\
\hline Neuquén & & & & & & \\
\hline Hectáreas & $8.564,00$ & $7.349,60$ & 6.793 .9 & $4.741,60$ & $1.758,10$ & $2.595,10$ \\
\hline Cantidad de Plantas & 3.275 .150 & 5.939 .902 & 2.420 .842 & 3.602 .009 & 849.401 & 2.321 .655 \\
\hline Plantas/Has & 382,4 & 808,2 & 356,3 & 759,7 & 483,1 & 894,6 \\
\hline
\end{tabular}

*Incluye fruta de membrillo

Fuente: INDEC, CNA 1988 Y 2002.

Del cuadro $\mathrm{N}^{\circ} 1$ extraemos como confirmación general el aumento de la productividad por hectárea en las dos provincias entre 1988 y 2002 . Aunque se registre una disminución de la superficie implantada en las explotaciones agropecuarias (EAP según el Censo Nacional Agropecuario), aumentó la cantidad de plantas con frutales de pepita, duplicando la cantidad plantas por hectáreas. En este sentido, la mejoría de la relación plantas por hectáreas es un indicador de reconversión tecnológicas en las unidades productivas relacionada con el cambio en la conducción de las plantaciones, pasando del sistema tradicional al sistema de conducción por espaldera o compacta. El aumento de la productividad por hectárea generó un aumento de los requerimientos de mano de obra durante los periodos de cosecha, incrementando de esta manera, la estacionalidad de la demanda. En lo que refiere a la cantidad de plantas por hectárea, las fuentes de Río Negro registran que el $70 \%$ del total de la superficie con frutales de pepita presentan una densidad superior a las 500 plantas por hectáreas (CAR, 2005). 
F. Svampa, Dinámicas socio-laborales en el Alto Valle de Río Negro.

Autoctonía. Revista de Ciencias Sociales e Historia, Vol. I, N²

Cuadro $\mathbf{N}^{\circ}$ 2. Distribución de la superficie implantada con manzanas y perales según sistema de conducción. Río Negro, 2005.\%

\begin{tabular}{|l|l|l|l|l|l|l|}
\hline \multirow{2}{*}{$\begin{array}{l}\text { Total de la provincia y } \\
\text { localidades }\end{array}$} & \multicolumn{4}{|l|}{ Manzana } & \multicolumn{2}{l|}{ Peral } \\
\cline { 2 - 7 } & Total & Espaldera & Tradicional & Total & Espaldera & Tradicional \\
\hline Total de Río Negro & $100 \%$ & $67,90 \%$ & $32,10 \%$ & $100 \%$ & $73,60 \%$ & $26,40 \%$ \\
\hline Alto Valle de Río Negro & $100 \%$ & $65,70 \%$ & $34,30 \%$ & $100 \%$ & $71,70 \%$ & $28,30 \%$ \\
\hline
\end{tabular}

Fuente: Secretaría de Fruticultura de Río Negro, CAR 2005

Río Negro concentra el $83 \%$ de la superficie implantada con frutales de pepita en la Patagonia Norte, el $82 \%$ de manzanas y el $85 \%$ de peras (CNA, 2002). Según el cuadro $\mathrm{N}^{\circ} 2$, el sistema de conducción más difundido para el 2005 era espaldera, tiene un peso superior en el total de la provincia, tanto en manzanas, como en peras que en las localidades del AVRN. Por otro lado, tiene mayor peso entre las plantaciones de peras que entre las plantaciones de manzana. Este fenómeno estaría relacionado con otro indicador de la modernización tecnológica, que es la antigüedad del monte frutal. La reconversión de un sistema de conducción significa desmontar las hectáreas existentes con sistema tradicional, invertir para adquirir nuevas plantas y esperar entre 4 o 5 años para las primeras cosechas.

Cuadro $\mathbf{n}^{\circ}$ 3. Cambios técnicos en el sector de empaque y frigorífico. Alto Valle de Río Negro

\begin{tabular}{|c|c|c|}
\hline Década & Cambio técnico & \\
\hline & Galpón de empaque & Frigorífico \\
\hline 1960 & $\begin{array}{l}\text {-Tratamiento de fruta para el ingreso } \\
\text { al frigorífico. } \\
\text {-Cajón bins. } \\
\text {-Autoelevador. }\end{array}$ & $\begin{array}{l}\text {-Masificación de la construcción de } \\
\text { frigoríficos. } \\
\text {-Utilización de productos químicos para la } \\
\text { conservación. }\end{array}$ \\
\hline 1970 & $\begin{array}{l}\text {-Caja telescópica } \\
\text {-Bandejas de pulpa moldeada } \\
\text { Hidrovaciado de bins y conducción de } \\
\text { agua. }\end{array}$ & $\begin{array}{l}\text {-Túneles de preenfriamiento y cámaras de } \\
\text { atmósfera controlada. }\end{array}$ \\
\hline 1980 & $\begin{array}{l}\text { Sector control de calidad de la fruta. } \\
\text { Mejoramiento del lavado de la fruta. }\end{array}$ & \\
\hline 1990 & $\begin{array}{l}\text {-Tamañadora electrónica. } \\
\text {-Llenadora automática de bins. } \\
\text {-Apilador automático de bins. } \\
\text {-Llenadora semiautomática. } \\
\text { Romaneo Electrónico. }\end{array}$ & \\
\hline
\end{tabular}

Fuente: Elaboración propia. 
F. Svampa, Dinámicas socio-laborales en el Alto Valle de Río Negro.

Autoctonía. Revista de Ciencias Sociales e Historia, Vol. I, N²

Los cambios tecnológicos mencionados en el cuadro $\mathrm{N}^{\circ} 3$ posibilitaron un mejor control de los ritmos de funcionamiento y la desestacionalización de las tareas en los galpones de empaque, sumado a la conservación de la fruta en frigoríficos, permitió procesar mayores volúmenes de fruta, evitando defectos técnicos. A partir de la década del '60, el acondicionamiento de la fruta durante todo el año, también posibilitó el incremento del volumen de trabajadores permanentes. Además, la vinculación del empaque con el frigorífico facilitó el control de la oferta de la fruta a la espera de mejores precios, manutención de la calidad de la fruta en los momentos de conflictos laborales y el control de los stocks de insumos. A partir de la década del '70, se refuerza la vinculación técnica de la unidad empaque-frío en un marco de mayores exigencias de calidad por el mercado. La aparición de nuevos materiales de trabajo fue reduciendo el nivel de habilidad requerida para la realización de algunas tareas como las del embalador de fruta en los galpones de empaque. La introducción de cajas de cartón cambió las formas de trabajo del personal encargado de la preparación del material de empaque y de embalaje. Hacia la década del '80, las empresas integradas poseían el 70\% de la capacidad instalada de frío (Merli y Bonifacio, 1996) y a comienzos de los '90 la aparición de tecnología electrónica comenzó a reemplazar partes mecánicas del proceso de trabajo.

\section{La actividad frutícola durante la post- convertibilidad}

Para inicios del siglo XXI, el sector agroindustrial en la Argentina, pero principalmente el que comercializaba la fruta al mercado externo esperaba la salida del cambio fijo. Cuando se dio este hecho a comienzos del 2002, en el AVRN, todos los agentes económicos de la cadena frutícola se encontraban en una situación de profundo compromiso financiero. En este marco, fueron los eslabones primarios de la cadena no integrados que vieron fuertemente amenazada su continuidad y factibilidad productiva, como así también los trabajadores asalariados. Los pequeños y medianos productores inferiores a 25ha, se encontraron en una situación de descapitalización, que se profundizó durante la época de la convertibilidad, por la nula rentabilidad que obtuvieron con la sobreevaluación del peso y el escaso crédito accesible.

Cuadro $\mathbf{n}^{\circ}$ 4. Caracterización de los productores según rango de tamaño y superficie bruta. AVRN. 2005.\%

\begin{tabular}{|l|l|l|l|l|}
\hline $\begin{array}{l}\text { Rango de } \\
\text { Tamaño (ha) }\end{array}$ & $\begin{array}{l}\text { Cant. De } \\
\text { productores }\end{array}$ & \%Productores & $\begin{array}{l}\text { Superficie } \\
\text { Bruta (ha) }\end{array}$ & $\begin{array}{l}\text { \% Superficie } \\
\text { Bruta (ha) }\end{array}$ \\
\hline $0-10$ & 1.545 & $49,80 \%$ & $8.267,43$ & $12,25 \%$ \\
\hline $10-25$ & 1.000 & 32,25 & $15.282,69$ & $22,65 \%$ \\
\hline $25-50$ & 333 & $10,7 \%$ & $11.453,35$ & $17,0 \%$ \\
\hline$>50$ & 222 & $7,2 \%$ & $32.468,31$ & $48,10 \%$ \\
\hline Total & $\mathbf{3 . 1 0 0}$ & $\mathbf{1 0 0 \%}$ & $\mathbf{6 7 . 4 7 1 , 7 8}$ & $\mathbf{1 0 0 \%}$ \\
\hline
\end{tabular}

Fuente: Elaboración propia a partir de los datos del CAR '05 facilitados por el INTA.

El cuadro $\mathrm{N}^{\circ} 4$ indica que el agrupamiento de la cantidad de productores según rango de tamaño disminuye a medida que aumenta la superficie de las unidades productivas, el $82,05 \%$ de un total de 3100 productores tiene bajo su control rangos de tamaño de superficie menores a 25 hectáreas para el AVRN. Se considera que la heterogeneidad de situaciones productivas en relación a la UOP da cuenta de la existencia en la estructura productiva frutícola en el AVRN de explotaciones tradicionales pequeñas que van de 5 ha a 10 ha con dedicación exclusiva del productor, fuerza de trabajo familiar, mano de obra transitoria durante la cosecha o para las actividades de poda. Luego están las explotaciones medianas de 10 a 25 ha en donde el productor y su familia realizan ciertos trabajos específicos y contrata mano de obra permanente y transitoria para las labores culturales, en particular la poda, el desmalezamiento, el raleo y la cura, y la cosecha. En las empresas grandes de 25ha en adelante, el productor tiende a limitarse a la administración, derivando las tareas de control de las chacras a un capataz. Se contrata mano de 
obra permanente para poder, limpiar acequias, el control de plagas y enfermedades ejecutados por un encargado tractorista y cosecheros para los períodos de cosecha. Luego de la devaluación del 2002 en la Argentina, los principales beneficiarios fueron las exportadoras de fruta que residían en el
AVRN, comenzando por las empresas de empaque integradas que frente al cambio de precios relativos, agregaron el aumento de los precios internacionales de la fruta fresca de contraestación (Rofman, 2006).

Cuadro $\mathbf{n}^{\circ}$ 5. Pluriactividad entre los productores (solo personas físicas), según actividad remunerada, sector y condición. AVRN. 2005

\begin{tabular}{|c|c|c|c|c|c|c|c|}
\hline \multicolumn{8}{|c|}{ Pluriactividad } \\
\hline 苞 & 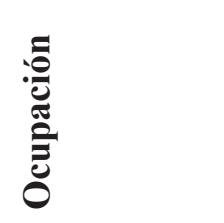 & 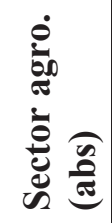 & 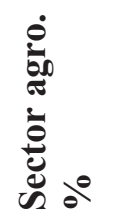 & 竭 & 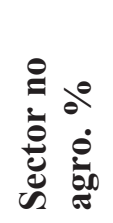 & $\stackrel{\bar{\sigma}}{\stackrel{\tilde{\theta}}{\theta}} \stackrel{0}{\sigma}$ & $\frac{e^{0}}{\frac{\sigma}{0}}$ \\
\hline \multirow[t]{3}{*}{ Si } & $\begin{array}{l}\text { Empleadores } \\
\text { y } \\
\text { trabajadores } \\
\text { cta. propia }\end{array}$ & 165 & $44,23 \%$ & 356 & 54,15 & 521 & $49,76 \%$ \\
\hline & $\begin{array}{l}\text { Asalariados } \\
\text { parte del año }\end{array}$ & 46 & $12,33 \%$ & 46 & 6,8 & 92 & $8,78 \%$ \\
\hline & $\begin{array}{l}\text { Asalariados } \\
\text { todo el año }\end{array}$ & 162 & $43,43 \%$ & 272 & 40,35 & 434 & $40,40 \%$ \\
\hline $\begin{array}{l}\text { Total } \\
\text { SI }\end{array}$ & & 373 & $100 \%$ & 674 & $100 \%$ & 1.071 & $100 \%$ \\
\hline $\begin{array}{l}\text { Total } \\
\text { No }\end{array}$ & & & & & & 1.541 & \\
\hline $\begin{array}{l}\text { Sin } \\
\text { datos }\end{array}$ & & & & & & 58 & \\
\hline $\begin{array}{l}\text { Total } \\
\text { General }\end{array}$ & & & & & & 2.646 & \\
\hline
\end{tabular}

Fuente: Elaboración propia a partir de los datos del CAR '05 facilitados por el INTA.

El cuadro $\mathrm{N}^{\circ} 5$ refleja que un $39,59 \%$ (1071 de los productores) tienen una actividad remunerada por fuera de la UOP en el AVRN, en relación a un 54,23\%. (1.541 los productores) que no tienen actividad remunerada por fuera de la UOP. Dicha situación obligaría a prestar atención a otras actividades remunerativas del Jefe/a de Hogar en la población rural y la pérdida de predominancia de la actividad agropecuaria como principal trabajo en la unidad familiar en la región. Dentro de los que tienen actividad remunerada un 35,62\% (373 productores) tienen actividad remuneradas en el sector agropecuario en relación a un $64,38 \%$ (674 productores) que tienen actividades remuneradas por fuera del sector agropecuario. Por otro lado, se registra una menor participación de los productores con actividad remunerativa en la categoría empleadores y trabajadores cuenta propia en el sector agropecuario $(44,23 \%)$ en relación al sector no agropecuario $(54,15 \%)$. Por su parte, la categoría de asalariado durante una parte del año presenta más participación el sector agropecuario (12,33\%) en relación al sector no agropecuario $(6,8 \%)$.

En este marco, el Estado viene cumpliendo un rol legitimador en la situación de la economía regional, 
F. Svampa, Dinámicas socio-laborales en el Alto Valle de Río Negro.

Autoctonía. Revista de Ciencias Sociales e Historia, Vol. I, N²

en tanto no ha intervenido en las relaciones desiguales, uno de ellos son los momentos de fijación de precios entre los productores independientes y las empresas comercializadoras e integradas. Estos últimos sacan ventaja de la situación de vulnerabilidad que caracteriza a los productores independientes de los estratos más endeble de la estructura frutihortícola. Además, el Estado no ha promovido políticas para superar las desigualdades estructurales en la actividad, como así también las responsabilidades de las empresas líderes. Más allá de las intenciones o limitaciones de las políticas públicas del Estado provincial, en este segmento la ausencia, insuficiencia de herramientas públicas precisas e integrales para la actividad y la debilidad de los planteos sectoriales de reconversión, dejan su impronta en un contexto de inconsistencia de experiencias asociativas y de persistencia de conductas individualistas derivadas del pensamiento de autosuficiencia que se vincula con la trayectoria histórica del pequeño productor (Landriscini et al., 2007).

Por otro lado, las características socio-económicas acerca de los productores frutícolas del AVRN no se dieron en el vacío, sino que se plasmaron en el territorio. El deterioro de la reproducción social del eslabón primario (Alvaro, 2013) dio como resultado, mejores condiciones para la entrada de otras actividades aún más desiguales y expoliadoras, que se van consolidando en la zona en paralelo los procesos de expansión urbana, incorporando superficie de la periferia a la ciudad; ésta avanza por los espacios rurales y áreas en producción rompiendo su estructura funcional. Cuando esos componentes avanzan sobre lo rural dan lugar a la conformación de dos entidades espaciales transicionales que se generan por la coexistencia en un mismo territorio de usos propios de lo urbano y lo rural: lo periurbano y lo rururbano. En lo que refiere a lo periurbano, actualmente domina la yuxtaposición de usos, conviviendo en tensión chacras de estratos que van de las 5 ha a 25 ha, con espacios urbanos, instalaciones y grandes equipamientos vinculados a la actividad hidrocarburífera4. Incluso galpones y talleres ubicados en la periferia urbana se han reconvertido de su original uso en la cadena frutícola a distintas actividades en el rubro de los hidrocarburos. Lo periurbano representa un espacio genérico que rodea a cualquier ciudad, mientras que en lo rururbano aunque presenta las mismas características, es la zona rural la que ejerce la predominancia, sin que estas pierdan sus atributos territoriales (económicos, socioculturales) como sucede en el periurbano. El concepto de rururbano -según la literatura- es una expresión que identifica a las áreas en tensión que rodean a las ciudades donde la presencia dominante de viviendas con hogares particulares unipersonales o multipersonales, dispersas y aisladas cohabitan con la persistencia de áreas agrícolas y forestales (o naturales). "Los procesos rururbanizadores del territorio generan transformaciones en el espacio, caracterizadas por un equilibrio inestable entre las funciones tradicionales y las nuevas funciones." (García Ramón, 1995: 53). Promoviendo el establecimiento de la población en zonas cercanas a las ciudades, facilitando el acceso a la infraestructura de servicios y refugio para las actividades en la época de baja demanda en la fruticultura; próximo a los lugares de fuerte demanda estaciona como las fincas y los empaques, típicamente rurales.

\section{El mercado de trabajo rural del AVRN}

Las estadísticas públicas oficiales de Argentina presentan dificultades para captar la complejidad de los mercados de trabajo agropecuarios y medir aspectos relacionados con el empleo en el sector y los trabajadores, estas cuestiones podrían resumirse en cierto sesgo netamente urbano industrial y de servicios en las formulaciones conceptuales. En este sentido, se puede pensar en la existencia de una contradicción entre la importancia del sector agropecuario en términos de exportación, de generación de divisas y de empleo, y las deficiencias de información estadísticas sólidas, regulares (Aguilera, 2007). Sin embargo, para el caso del AVRN, es posible analizar ciertos aspectos del mercado de trabajo a partir del EPH que surgió en la Argentina en la década del '70 y su marco de referencia fue el Censo Nacional de Población de 1970. Dicha encuesta, a partir de 1972 fue agregando los diferentes aglomerados urbanos y que en la actualidad llegan a 31. El aglomerado Alto Valle de Río Negro se incorporó al relevamiento en septiembre de 1977 con el objetivo de lograr una cobertura del ámbito rural. En este caso, para el aglomerado rural-urbano Alto Valle de Río Negro, el objeto de estudio no fue solamente la población urbana del aglomerado en el mercado de trabajo, sino una unidad que concibiera la existencia de un continuo rural-urbano. Esto se debe al impacto de la producción primaria, en donde los datos analizados no comprenden a todos los 
trabajadores rurales, sino sólo a los comprendidos en áreas periurbanas. El EPH del AVRN sufrió diversos ajustes de todos los temas relevados. En primer lugar, las categorías ocupacionales clasificatorias empleadas tenían un limitante teórico para dar cuenta de la diversidad causada por el fenómeno de la estacionalidad (Aguilera, et al. 2015). En este sentido, en la definición de la condición de actividad se incluyó el recate del grupo de los inactivos "disponibles", entendidos como la población que se "refugia en la inactividad" durante los períodos de receso. De esta forma se agrega a las tres categorías ocupaciones (ocupados, desocupados e inactivos) la categoría de los "disponibles". Otro aspecto operativo a resaltar es el tratamiento a la variable "Rama de actividad económica del establecimiento" que hasta el 2011 utilizó la codificación a tres dígitos de la CIIU tercera revisión(5 para identificar las ramas de producción (Galpón de empaque). A continuación, se comparte una lectura de los principales indicadores del mercado de trabajo del aglomerado rural urbano Alto Valle de Río Negro a partir de las ondas (de marzo y septiembre) desde 1995 al 2011. Los siguientes indicadores permiten ofrecer un marco para estimar en qué forma el ciclo de la fruticultura repercute en el empleo y en la participación de la población local

en el mercado de trabajo.

Cuadro $\mathbf{n}^{\circ}$ 6. Distribución de la población total, de 14 años y más y PEA por condición migratoria y sexo para el aglomerado rural-urbano AVRN. Diferencia absoluta en puntos porcentuales.

\begin{tabular}{|c|c|c|c|c|c|c|c|c|c|c|c|c|c|c|}
\hline \multirow[t]{2}{*}{ 官 } & \multicolumn{4}{|r|}{$\begin{array}{l}\dot{0} \overline{0} \\
\stackrel{5}{0} \\
\stackrel{0}{0}\end{array}$} & \multirow[t]{2}{*}{$\stackrel{\circ}{\circ}$} & \multirow[t]{2}{*}{$\stackrel{\partial}{\circ}=$} & \multirow[t]{2}{*}{ 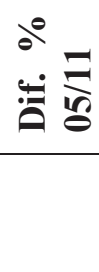 } & \multicolumn{4}{|r|}{$\frac{\pi}{2}$} & \multirow[t]{2}{*}{ 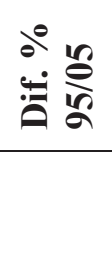 } & \multirow[t]{2}{*}{$\stackrel{\partial}{\stackrel{\partial}{a}}=$} & \multirow[t]{2}{*}{$\stackrel{\circ}{\stackrel{\circ}{\sigma}}$} \\
\hline & مू & ஜ̊ & $\stackrel{\infty}{\stackrel{\text { }}{~}}$ & $\overline{\bar{~}}$ & & & & ڤ̊ & ஜ̊ & $\stackrel{\infty}{\stackrel{\text { }}{~}}$ & $\overline{\bar{\nu}}$ & & & \\
\hline $\begin{array}{l}\text { No } \\
\text { migrante }\end{array}$ & 51,7 & 56,8 & 58,7 & 51,6 & 5,1 & 0,1 & 5,2 & 36,2 & 44,8 & 49,1 & 37,9 & 8,6 & 1,7 & 6,8 \\
\hline Varón & 25,3 & 26,9 & 28 & 28,7 & 1 & 3,4 & 1,8 & 21,5 & 28,5 & 28,7 & 23,1 & 7 & 1,6 & 5,4 \\
\hline Mujer & 26,5 & 29,9 & 30,8 & 23 & 3,5 & 3,5 & 7 & 14,7 & 16,3 & 20,4 & 14,9 & 1,6 & 0,2 & 1,4 \\
\hline $\begin{array}{l}\text { Mig. } \\
\text { Interno }\end{array}$ & 37 & 33,6 & 28,6 & 41,4 & 3,4 & 4,4 & 7,8 & 48,8 & 43,9 & 36,2 & 53,9 & 4,9 & 5 & 9,9 \\
\hline Varón & 17,7 & 15,3 & 13,5 & 28,8 & 2,4 & 11,1 & 13,5 & 29,8 & 26,3 & 21,8 & 44,5 & 3,5 & 14,7 & 18,2 \\
\hline Mujer & 19,3 & 18,3 & 15 & 12,6 & 1 & 6,7 & 5,7 & 19,1 & 17,7 & 14,3 & 9,4 & 1,4 & 9,7 & 8,3 \\
\hline $\begin{array}{l}\text { Mig. } \\
\text { Externo }\end{array}$ & 11,3 & 9,5 & 12,7 & 7 & 1,7 & 4,3 & 2,5 & 14,9 & 11,3 & 14,8 & 8,2 & 3,6 & 6,7 & 3,1 \\
\hline Varón & 5,4 & 4,4 & 5,9 & 3,4 & 1 & 2 & 1 & 8,9 & 7,3 & 9,5 & 5,4 & 1,6 & 3,6 & 2 \\
\hline Mujer & 5,9 & 5,1 & 6,8 & 3,6 & 0,8 & 2,3 & 1,5 & 6 & 4 & 5,3 & 2,8 & 2 & 3,2 & 1,2 \\
\hline Total & 100 & 100 & 100 & 100 & & & & 100 & 100 & 100 & 100 & & & \\
\hline
\end{tabular}

*Coeficiente de variación mayor a 10\%

Fuente: Elaboración propia a partir de las bases de EPH de la DGEC-INDEC 
F. Svampa, Dinámicas socio-laborales en el Alto Valle de Río Negro.

Autoctonía. Revista de Ciencias Sociales e Historia, Vol. I, N²

Como se observa en el cuadro $\mathrm{N}^{\circ} 6$, se registra una disminución de 5,1 pp. de la población migrante externa en la población del AVRN desde 1995 al 2005, con un repunte de $1,9 \mathrm{pp}$ en el 2008 para migrantes externos. Por su parte, la migración interna cayó 4,4 pp. 1995-2008 y repuntó notablemente en el 2011 con $12,8 \mathrm{pp}$. de diferencia en relación al 2008. Podría pensarse en este sentido, que la zona a partir de 1995 perdió capacidad de atracción y absorción de población joven para migrantes externos, en un marco de retracción de la actividad frutícola hasta mediados del 2003, crecimiento del dinamismo de cultivos de exportación de Chile, restricciones implementadas por el estado argentino en la incorporación de trabajadores extranjeros y la disminución del salario real en Argentina (Radonich, Steimbreger, Ozino Caligaris, 1999a; Aguilera 2007). El proceso de crisis y reestructuración que se registró en ochenta en el territorio valletano generó un aumento cuantitativo de la producción y una profundización del proceso de acumulación capitalista. Esta tendencia condujo en los noventa a remarcar la brecha entre los distintos actores sociales en función de las nuevas características que asumió la producción en el contexto de una economía globalizada (Radonich, 2001), reforzándose la situación a través de los complejos agroindustriales. Estos procesos provocaron, la caída de la figura del asalariado permanente tradicional y por otro incorporaron asalariados permanentes, ligados a la empresa agrícola modernizada, con un mercado de trabajo local autosuficiente, donde la población migrante limítrofe e interna se encuentra establecida y envejecida (Aguilera, 2007). Por otro lado, la población no migrante del AVRN registró un aumento considerable entre 1995 y el 2008 con una diferencia de 7 pp. El aumento entre 1995 y el 2008 estaría refiriendo a los migrantes que se establecieron en forma definitiva en la zona, formaron sus familias y continuaron con el ciclo reproductivo, es decir, su descendencia paso a ser parte de la población no migrante. En este sentido al observar para 1995-2005 los 5,1 pp. Que crece la población no migrante en la población total, esta categoría llega a $8,6 \mathrm{pp}$. en la PEA. El AVRN no recibe niños o población de hasta 14 años como migrantes, por lo tanto, el menor arribo de población migrante externa de Chile y desde el interior del país desde 1995 al 2008 es una evidencia de que en la zona se ha constituido un asentamiento definitivo (Aguilera, 2007).

Cuadro $\mathbf{n}^{\circ}$ 7. Evolución de las principales tasas del mercado para el aglomerado rural-urbano AVRN. 19952008

\begin{tabular}{|l|l|l|l|l|l|l|l|l|l|l|l|l|}
\hline Tasas & mar & sep- & & sep- & & sep- & & sep- & & sep- & & sep- \\
\hline Actividad6 & 42,4 & 30,5 & 41,5 & 39,8 & 40,6 & 37,9 & 41,8 & 39,8 & 40,6 & 40,1 & 44,5 & 43,5 \\
\hline Desempleo 7 & 7,9 & 14,1 & 8,4 & 11,5 & 13 & 15,6 & 6,4 & 9,6 & 3,7 & 6,3 & 3,3 & 4,5 \\
\hline Ocupación_ & 37 & 35,6 & 39,1 & 36,2 & 38,4 & 32 & 39,1 & 36,4 & 37,6 & 40,8 & 41,5 & 39,6 \\
\hline
\end{tabular}

Fuente: Elaboración propia a partir de las bases de EPH de la DGEC-INDEC

Según el cuadro $\mathrm{N}^{\circ}$ 7, la tasa de actividad en el AVRN ha sido a lo largo de la historia mayor durante los meses cosecha desde enero-abril (el EPH lo registraba en marzo), por lo que no debería sorprender que a partir de 2004 la participación de la población en el mercado de trabajo haya crecido más en marzo. Por otro lado, entre 2004 y 2008 la participación de la población en el mercado de trabajo no varió demasiado entre un mes y otro; sus diferencias tendieron a atenuarse, lo que estaría dando cuenta que la actividad frutícola fue mucho más determinante en la evolución de la tasa de actividad en los años 1995-2002 que durante el lapso 2004-2008.
En este marco es notorio el descenso de la desocupación y el crecimiento del empleo en general a partir del 2004, pero el comportamiento de estos indicadores es particularmente destacable en el mes de septiembre, durante el receso de la actividad frutícola, en donde las principales actividades son la poda y limpieza de acequias, control de plagas, laboreos del suelo y otras prácticas culturales que requieren de menos demanda de trabajadores rurales. Si tomáramos el período 1995-2008 como aparecen confirmamos nuevamente que las tasas de desocupación para el aglomerado Alto Valle de Río Negro son mayores en septiembre que en marzo, es 
F. Svampa, Dinámicas socio-laborales en el Alto Valle de Río Negro.

Autoctonía. Revista de Ciencias Sociales e Historia, Vol. I, N²

decir en los períodos de contracosecha, con tasas de desocupación menores a dos dígitos a partir de marzo del 2004. El impacto de la población disponible en la desocupación es bastante notorio lo que podría concebirse como refugio en la inactividad durante las épocas de receso, producto del desaliento por la búsqueda constante de empleo entre las personas que conocen los ciclos de actividad en el año (Aguilera, 2007).

Cuadro $\mathbf{n}^{\circ}$ 8: Tasa de actividad de la población 14 años y más por sexo en el aglomerado rural-urbano AVRN, según ondas. 1995-2005.

\begin{tabular}{|l|l|l|l|}
\hline Ondas & Total & Varones & Mujeres \\
\hline Marzo 1995 & & & \\
\hline Setiembre 1995 & $\mathbf{4 2 , 4}$ & 75,0 & 46,0 \\
\hline Marzo 1999 & $\mathbf{3 0 , 5}$ & 72,6 & 44,8 \\
\hline Setiembre 2004 & $\mathbf{4 1 . 5}$ & 77,5 & 45.5 \\
\hline Marzo 2005 & $\mathbf{3 9 , 8}$ & 71,5 & 37,1 \\
\hline
\end{tabular}

Fuente: Elaboración propia a partir de las bases EPH de la DGEC-INDEC

Por otro lado, según el cuadro $\mathrm{N}^{\circ} 8$, las fluctuaciones de las tasas de actividad según sexo en los totales vuelven a confirmar los ciclos de estacionalidad de la actividad frutícola en la zona, marcando un alza de la misma en los períodos de cosecha hasta marzo cuando se hacía el primer relevamiento. Los varones presentan tasas de actividad mayores en marzo de 1995, 1999 y el 2005 que en septiembre de 1995 y 2004 . Las mujeres presentan fluctuaciones menores ligadas a los ciclos de la actividad frutícola con una fuerte disminución en marzo del 2004 en comparación a marzo de 1995, lo cual estaría dando cuenta que la participación de las mujeres está ligada a los avatares económicos del contexto. Cuando la ocupación o el ingreso aportado por los varones no se encuentran garantizados por una crisis macroeconómica, regional o sectorial, es una estrategia de los hogares la entrada al mercado de otros miembros del hogar.
Por otro lado, aunque no se cuenta con datos actuales, en un marco de aumento de la escolarización, la fiscalización y el control sanitario; el fenómeno del trabajo infantil y adolescente sigue persistiendo en los hogares de pequeños productores como en trabajadores asalariados en el AVRN. Según datos de la Encuesta de Actividades de Niños, Niñas y Adolescentes para el 2004 en Río Negrog, el 8,7\% del total de niños/as trabajan en tareas vinculadas al sector primario, los cuales se distribuyen en hogares de asalariados permanentes y trabajadores por cuenta propia. La ocupación temporaria tiende a ser una de las formas más difundidas a partir de la cual se produce la entrada de jóvenes como trabajadores asalariados y realizan aportes económicos en la familia (Neiman, 2012). 
F. Svampa, Dinámicas socio-laborales en el Alto Valle de Río Negro.

Autoctonía. Revista de Ciencias Sociales e Historia, Vol. I, N²

Cuadro $\mathbf{n}^{\circ}$ 9. Tasa de desocupación según género para el aglomerado rural-urbano AVRN. Ondas de 2004,2005, 2008.

\begin{tabular}{|c|c|c|}
\hline \multicolumn{3}{|c|}{$\begin{array}{l}\text { Tasa de desempleo según } \\
\text { género }\end{array}$} \\
\hline Meses & Varones & Mujeres \\
\hline mar-04 & 5,9 & 7,5 \\
\hline sep-04 & 9 & 9,2 \\
\hline mar-05 & 3 & 3,9 \\
\hline sep-05 & 6 & 7,9 \\
\hline mar-08 & 1,7 & 1,9 \\
\hline sep-08 & 5,5 & 5,6 \\
\hline
\end{tabular}

Fuente: Elaboración propia a partir de distintas ondas de EPH-DGEC-INDEC

Si nos atenemos a la tasa de desocupación según género del cuadro $\mathrm{N}^{\circ}$, desde el 2004 se registra a ritmos constantes una disminución de la tasa de desocupación masculina, lo cual da cuenta de una mejoría en términos de generación de empleo, pero con la persistencia de índices de desempleo alto en septiembre. Por otro lado, aunque las mujeres presentan índices de desocupación que decrecen, su descenso muestra un comportamiento más fluctuante en comparación a los varones. Si comparamos los índices de septiembre tanto para ambos géneros, se vislumbra una disminución continua de la desocupación en épocas de caída de actividad frutícola. Brizuela y Tumini (2008) analizan que el mercado de trabajo en Argentina mostraba una notoria inequidad de género ya hacia el año 2007. Señalan además que la recuperación económica iniciada en 2003 benefició más a los hombres que a las mujeres por el crecimiento de actividades económicas vinculadas históricamente al empleo masculino, lo que conllevó en una menor reducción del desempleo femenino.

\section{Fragmentaciónjurídicay representación sindical}

La introducción de una variedad de exigencias relacionadas a la calificación para la recolección y el acondicionamiento de la fruta se contrapone con la continua persistencia de condiciones de vida y formas vulnerables de contratación dada la estacionalidad del trabajo (poda, raleo, recolección), a la que se agregan formas de elusión/evasión de la formalización. Más allá de que la Comisión Nacional de Trabajo Agrario (CNTA) ha cobrado impulso con la Ley $\mathrm{N}^{\circ}$ 26.727/11 -sancionada en diciembre de 2011 y reglamentado en marzo de 2013-la situación de los trabajadores temporarios sigue siendo muy endeble10. En este sentido, la fragmentación jurídica ha sido una problemática constante entre los diferentes trabajadores de la actividad frutihortícola, profundizando segmentación de los programas institucionales en materia sindical y de organismos de control. Esta problemática se expresa en el hecho de que existen trabajadores excluidos del régimen de trabajo agrícola de la ley 26.727/11, quedan bajo la aplicación de Convenios Colectivos de Trabajo (CCT) "como sucede en los trabajadores de fruta (CCT 1/76 y la Ley 20.744" (Daniela y Sánchez, 2016: 27). Por lo tanto, en estos casos, la norma subsidiaria no es la Ley 26.727 sino la Ley de Contrato de Trabajo (LCT) y quedan exentos de ciertos beneficios, atentando de esta manera contra el principio jurídico de igualdad ante la ley en condiciones similares.

De este modo, el nuevo régimen de trabajo agrario no engloba bajo un mismo régimen normativo al empleo rural temporario: esto aparece en el artículo 7 donde establece cuales son los trabajadores incluidos11; por otro lado, los trabajadores excluidos aparecen en el artículo 3.12 Lo s trabajadores de cosecha de frutas en el AVRN quedan comprendidos por CCT especiales, como el CCT $\mathrm{N}^{\circ} 1 / 76$ que corresponde a la cosecha de manzana en Río Negro y Neuquén. 
F. Svampa, Dinámicas socio-laborales en el Alto Valle de Río Negro.

Autoctonía. Revista de Ciencias Sociales e Historia, Vol. I, N²

En este sentido, los trabajadores de cosecha, luego de haber terminado la cosecha y se trasladan a otra actividad (sector de empaque o frigorífico) transita distintos registros y es regulado por legislaciones diferentes a pesar de tratarse de la misma actividad rural.

Este hecho resulta ser una complicación para la participación gremial de los trabajadores, e incide de forma negativa en las posibilidades de reclamo y exigencias de derechos.

\section{Reflexiones finales}

El agro argentino se ha desarrollado de manera heterogénea en las distintas regiones productivas, ya que en cada lugar el capitalismo se instaló y expandió bajo formas diferentes. Los cultivos de la fruticultura que requieren de inversión de capitales fijos funcionan en las etapas de expansión como colonizadores del espacio geográfico, requieren de infraestructura en espacios de baja densidad poblacional, atrayendo migrantes y en momentos de consolidación favorecen el asentamiento de la mano de obra. Este proceso fue característico del AVRN. La consolidación del modo de producción capitalista en esta región se logró facilitando el asentamiento urbano o periurbano de la mano de obra asalariada necesaria para la producción de frutas. Avanzado el siglo XX y en la década del '90 en el marco de la globalización, los gobiernos pusieron en marcha una forma de concebir el espacio geográfico nacional que desmanteló la red de regulaciones que aseguraban un lugar de consideración a las economías regionales en el proceso de desarrollo nacionales. Además, en un marco de expansión horizontal de las ciudades, se van consolidando usos y actividades de tipo urbano sobre usos rurales, provocando afectaciones ambientales y en el uso de la infraestructura, cambios en la renta del suelo (de rentas agrarias a rentas urbanas en función de alcanzar rápidos beneficios), así como conflictos por la presión que ejerce un uso del territorio sobre otro. Aunque a partir del 2004 los indicadores laborales para el AVRN reflejaron cierto mejoramiento en relación al período 1995-2005, las consecuencias de la globalización los sistemas de producción agroalimentarias iniciados a fines de los '80 parecen haber quebrado con el esquema de inversión productiva, configurando un paisaje social caracterizado por la fuerte diferenciación y heterogeneidad de los diversos sujetos sociales, la pérdida de posición y de capacidad de negociación de los productores independientes y de los trabajadores rurales. Los procesos que abarcan la producción, distribución y el consumo están inducidos por cambios técnicos a escala de la fruticultura que ha condicionado a los estratos más endebles de la cadena, aquellos históricamente vinculados a los productores familiares, al punto tal de producir un impacto heterogeneizador productivo y laboral en un marco de constante modernización tecnológica en cuanto a escala, productividad, producción y comercialización entre los distintos estratos de productores con mayor o menor grado de integración horizontal y vertical hacia adelante.

La demanda laboral en la producción agrícola se ha desestacionalizado parcialmente, conformándose trabajadores con mayor continuidad, calificación y polivalencia. Los temporarios de chacra, por otro lado, son demandados en períodos más largos y su volumen crece con el aumento de los rendimientos y de la superficie implantada, aunque también se les requiere mayores destrezas. En este sentido, Galafassi remarca acerca de la "especificidad del proceso de trabajo rural donde el mercado de trabajo se corresponde con la alta heterogeneidad del sector, producida por sus vinculaciones con diversos procesos: industrialización diferencial de la agricultura (tanto en sus articulaciones hacia atrás como hacia adelante), aumento de interacciones con lo urbano, y la coexistencia de distintas estrategias y modalidades de organización de la producción, aún para un mismo rubro o producto." (Galafassi,2002: 3). Como última reflexión y tarea pendiente, en una zona caracterizada por una marcada especialización en el cultivo intensivo de peras y manzanas, resulta de interés indagar para futuras investigaciones la tensión por el uso del espacio rural, debido a la incidencia de nuevas actividades no agropecuarias en el área; cabe dar cuenta de cómo una serie de transformaciones que se van dando en la actividad frutihortícola van haciendo lugar a la llegada de actividades extraagrarias, ligadas a la construcción residencial y los servicios, así como otras -fuertemente cuestionadascomo sucede en la actualidad con la proliferación de la actividad petrolera, orientada a la exploración y explotación de hidrocarburos no convencionales con el empleo del sistema técnico de fractura hidráulica múltiple. 
F. Svampa, Dinámicas socio-laborales en el Alto Valle de Río Negro.

Autoctonía. Revista de Ciencias Sociales e Historia, Vol. I, N²

\section{Bibliografía}

Aguilera, M. E. (2007): ¿Se van para volver? Trabajadores migrantes y mercado de trabajo en el Alto Valle del Río Negro. 1995-2005. Argentina. (Inédito), Tesis de Maestría en Demografía Social, Universidad Nacional de Luján.

Aguilera, M.E; Crovetto, M.MyEjarque, M(2015): “Los mercados de trabajo agropecuarios en Argentina. Un proceso de diseño de estrategias metodológicas para captar un objeto complejo". Presentado en Revista Latinoamericana de Metodología de la Investigación Social, 9, pp. 67-83

Alvaro, M. B. (2008): Estrategias de reproducción social de los productores chacareros. Revista de la Universidad del Comahue, 14, pp 29-52. [http:// fadeweb.uncoma.edu.ar/extension/publifadecs/ revista/revista14/05-Alvaro.pdf]

Alvaro, M. B. (2013): Estrategias de reproducción social en la producción familiar capitalizada, los chacareros del Alto Valle de Rio Negro. Buenos Aires: Editorial La Colmena.

Bandieri, S. y G. Blanco, (1994): "Comportamiento histórico del subsistema frutícola regional". En G. De Jong et al; El Minifundio en el Alto Valle del Río Negro: estrategias de adaptación. Neuquén. Universidad Nacional del Comahue. Facultad de Ciencias Agrarias y Facultad de Humanidades, pp.1944.

Bartolomé, L. (2000): Los colonos de Apóstoles. Estrategias adaptativas y etnicidad en una colonia eslava de Misiones. Posadas: Editorial Universitaria

Bendini, M. y C. Pescio (1996): Panorama y perspectivas del trabajo frutícola. En Bendini, M. y C. Pescio, coord., Trabajo y Cambio Técnico. El caso de la agroindustria frutícola en el Alto Valle. GESA. Buenos Aires. Editorial La Colmena

Bendini, M. y P. Tsakoumagkos, coords. (1999): Transformaciones agroindustriales y laborales en nuevas y tradicionales zonas frutícolas del Norte de la Patagonia. Cuaderno GESA N ${ }^{\circ} 3$ - PIEA N 10. Buenos Aires. FCE-UBA.

Bendini, M. y P. Tsakoumagkos (2002): "Regiones agroexportadoras, complejos agroalimentarios y producción familiar. Controles y resistencias". En Revista Realidad Económica, 190. Instituto Argentino para el Desarrollo Económico, pp. 116-133.
Bendini, M. y P. Tsakoumagkos (2003): "El agro regional y los estudios sociales. Temáticas y reflexiones". En Bendini, M. et al (org.): El campo en la Sociología actual. Buenos Aires: Editorial La Colmena.

Bendini, M. y P. Tsakoumagkos (2004): "Consideraciones generacionales sobre los chacareros de la cuenca de río Negro". En Bendini, M. y C. Alemany, Crianceros y Chacareros en la Patagonia. Cuaderno GESA N ${ }^{\circ}$. Buenos Aires: Editorial La Colmena, pp. 116-133.

Bendini, M.y P.Tsakoumagkos (2007): "Pluriactividad en los chacareros del Alto Valle. Análisis de datos secundarios en dos zonas tradicionales de la fruticultura en Río Negro, Argentina”. Presentado en las $\mathrm{V}$ Jornadas Interdisciplinarias de Estudios Agrarios y Agroindustriales. FCE-UBA. Buenos Aires.

Blanco, G. (1999): "La historia de un origen pionero, un pasado de gloria y un presente difícil" en INTA: Fruticultura moderna: tecnología, transferencia, capacitación, organización. Nueve años de Cooperación Técnica. INTA-GTZ. Alto Valle de Río Negro y Neuquén, pp.21.

CEIL/PISPAL. (1982): "Evolución del Empleo agropecuario en la ARGENTINA, 1914-1969”. Vol.II. (Inédito).

De Jong, G. (2010): La fruticultura patagónica del Alto Valle. Buenos Aires: Editorial La Colmena.

Doeswijk, A. et al (1998): Juntando recuerdos en oro. Municipalidad de Fernández Oro, Río Negro. Neuquén. Editorial de la Universidad Nacional del Comahue.

García Ramón y María Dolors (1995): Geografía rural. Madrid. Editorial Síntesis.

Galafassi, G. (2002): "Reestructuración productiva, organización del proceso de trabajo y manejo de tecnologías: Un estudio de caso en la producción frutícola y forestal", Mundo Agrario, Revista de Estudios Rurales, 2 (4), 24 pp.

INDEC. (1988): Censo Nacional Agropecuario 1988. Resultados generales. Provincias de: Río Negro y Neuquén. Disponible en [http://www.indec.gov.ar]

INDEC. (2001): Censo Nacional Agropecuario 2002. Resultados definitivos. Provincias: Río Negro y Neuquén. Disponible en: [http://www.indec.gov.ar] 
Landriscini, G. (2001): "El caso de la fruticultura: metamorfosis en la división y organización del trabajo. Del "distrito" al "sistema de empresas" $\mathrm{y}$ al "sistema institucional territorial". Ponencia presentada en Segundas Jornadas Interdisciplinarias de Estudios Agrarios y Agroindustriales. F.C.E. U.B.A. noviembre de 2001.

Merlo, R. y J. L. Bonifacio (1996): El cambio tecnológico en la fruticultura del Alto Valle. En Bendini M. y C. Pescio (Coordinadores). Trabajo y Cambio Técnico. El caso de la agroindustria frutícola en el Alto Valle. GESA. Buenos Aires. Editorial La Colmena

Miranda, O. (1995): "El cambio técnico en la Agricultura Familiar del Alto Valle de Río Negro". Revista RURALIA, 6 (1), pp. 28-46.

Miranda, O. (1997): “Organización del trabajo y acumulación de capacidades tecnológicas: una aproximación desde la fruticultura familiar". En Estudios del trabajo $\mathrm{N}^{\circ}$ 14. Segundo semestre de 1997. IADE. Buenos Aires, pp. 115-136

Neiman, G. (2012). Caso de Argentina. En Políticas de mercado de trabajo y pobreza rural en América Latina. Soto Baquero, F. y Klein, E. (Coord.) Vol II. pp. 10-35. Disponible en: http://www.fao.org/3/a-i2644s. pdf

Radonich, M., Steimbreger, N. y Ozino, C.M.S. (1999): "Expansión productiva y espacial de grandes empresas frutícolas de norpatagonia”. Ponencia presentada en Primeras Jornadas Interdisciplinarias de Estudios Agrarios y Agroindustriales. F.C.E. U.B.A. Noviembre de 1999.

Reguera, A. (2007): "Las tensiones de nuestra historia. Formas de concebir el desarrollo del Capitalismo en América Latina", en Graciano, O. y S. Lázzaro, comp., La Argentina Rural del Siglo XX. Fuentes, problemas y métodos. Buenos Aires: Editorial La Colmena, pp. 33-39.

Rofman, A. (2005): "La pequeña producción rural y la cadena frutícola en el Valle de río Negro hacia fines de la década de los '90”, Realidad Económica. IADE, 213, pp. 52-75.

Sacroisky, Ariana. (2003): "La producción de manzanas en el Alto Valle de Río Negro y Neuquén: Comprendiendoladécadadel'90apartirdeunanálisis estructural". En Terceras Jornadas Interdisciplinarias de Estudios Agrarios y Agroindustriales. F.C.E. U.B.A. 5 al 7 de noviembre de 2003.
Sánchez, D. (2016): "Trabajo rural temporario y fragmentación normativa en Argentina". Revista Pilquen. 19 (2), pp 25-37

Secretaría de Fruticultura de Río Negro. (2005): "Censo Provincial de Agricultura bajo Riego. CAR 2005". http://www.fruticultura.rionegro.gov.ar/ index.php?contID $=8140$

Recibido 10 de abril de 2017. Aceptado: 7 de julio de 2017.

\section{Notas}

$1 \quad$ El AVRN comprende a los Municipios de Allen (estudio de caso para este trabajo), Campo Grande, Cervantes, Chichinales, Cinco Saltos, Cipolletti, Contralmirante Cordero, Gral. Fdez. Oro, Gral. Enrique Godoy, Gral. Roca, Ingeniero Huergo, Mainque y Villa Regina.

2 También podemos considerar la aparición de la pulverizadora en 1970, el riego por aspersión para el control de heladas, el uso de fertilizantes químicos, control de plagas, raleo químico y el uso de herbicidas a partir de 1980.

3 El sistema más moderno de conducción por espaldera se basa en la colocación de árboles en hileras guiados sobre los cuales las ramas son conducidas horizontalmente. Por medio de este sistema los árboles poseen un menor porte y follaje, facilitando las prácticas culturales y mejorando la calidad de la fruta, permitiendo una productividad de hasta 50 toneladas por hectárea. Esta tecnología al requerir de gran cantidad de inversión inicial, resulta inaccesible para gran parte de los pequeños productores.

$4 \quad$ En la ciudad de Allen, ubicado en el Departamento de General Roca, en la provincia de Río Negro -Argentina- si bien se observa una alternancia entre la estructura rural y la urbana, las actividades frutícolas aún mantienen una cierta importancia. En el área es posible hallar población rural dispersa (que reside en campo abierto), población aglomerada (en núcleos construidos en torno a la estación ferroviaria) y población urbana. Se parte de una situación propia de áreas rururbanas en las que se manifiestan situaciones conflictivas que atañen a distintas funciones: áreas residenciales de baja densidad, actividades económicas ligadas a la matriz frutícola de la región y desde el 2012 hasta la fecha, la expansión de la frontera hidrocarburífera con la explotación de hidrocarburos no convencionales sobre predios rurales, impulsado por YSUR, subsidiaria de YPF. Clasificación Industrial Internacional Uniforme de 1989.

$6 \quad$ Está integrado por las personas que tienen una ocupación o que sin tenerla la están buscando activamente. Está compuesta por la población ocupada más la población desocupada. El cálculo de la tasa de actividad se obtiene a partir del porcentaje entre la población económicamente activa y la población total. 
7 Se refiere a personas que, no teniendo ocupación, están buscando activamente trabajo y están disponibles para empezar a trabajar. El cálculo de la tasa de desocupación se obtiene a partir del porcentaje entre la población desocupada y la población económicamente activa.

$8 \quad$ Conjunto de personas que tiene por lo menos una ocupación, es decir que en la semana de referencia ha trabajado como mínimo una hora (en una actividad económica). El cálculo de la tasa de desocupación se obtiene a partir del porcentaje entre la población ocupada y la población total.

9 La encuesta fue llevada a cabo a través del Ministerio de Trabajo, Empleo y Seguridad Social junto con el Instituto Nacional de Estadísticas y Censos.

10 La nueva ley transfirió un conjunto de funciones del registro Nacional de Trabajadores Agrícolas (RENATRE) al nuevo Registro Nacional de Empleadores y Trabajadores Agrícolas (RENATEA). Esta nueva entidad autárquica tiene facultades de inspección en referencia a la registración laboral de los trabajadores rurales y el control del cumplimiento de los empleadores de la Ley 25.191.

11

Ley 26.727, Art. 7: "Trabajadores rurales incluidos son: a) Manipulación y almacenamiento de cereales, oleaginosos, legumbres, hortalizas, semillas u otros frutos o productos agrarios, excepto que se realicen en establecimientos industriales. b) Tareas prestadas en ferias y remates de hacienda y c) Empaque de frutos y productos agrarios propios".

12 Ley 26.727, Art. 3: “Trabajadores rurales excluidos: a) Personal afectado principalmente a actividades industriales, comerciales, turísticas, de transporte o servicios, aunque se desarrollaren en empresas o establecimientos mixtos, agroindustriales o agrario-comerciales o de cualquier otra índole. b) Trabajador contratado para tareas ajenas a la actividad agraria. c) Trabajador del servicio doméstico. d) Personal administrativo. e) Personal dependiente del estado nacional, provincial o municipal. f) Al trabajador ocupado en tareas de cosecha y/o empaque de frutas, el que se regirá por la ley LCT $20.744 \mathrm{~g}$ ) Trabajadores comprendidos en convenciones colectivas de trabajo con relación a las actividades agrarias incluidas en el régimen de negociación". 